\title{
New Ex-vivo Organ Model for Percutaneous Renal Surgery
}

\author{
Florian Imkamp, Christoph von Klot, Udo Nagele, Thomas R.W. Herrmann \\ Clinic for Urology and Urologic Oncology (FI, CvK, TRWH), Hannover Medical School, Hannover, \\ Germany and Department of Urology, General Public Hospital (UN), Hall in Tirol, Austria
}

\begin{abstract}
Objectives: Percutaneous Renal Surgery (PRS) is a demanding procedure and success is mostly hampered by the lacking of training facilities. Thus, the purpose of the study was to evaluate a significantly improved pre-existing porcine kidneytraining model for percutaneous renal access and PRS.

Materials and Methods: A biologic training model using porcine kidneys coated by a full-thickness porcine skin flap was prepared. The ureter was dissected, stones were placed into the collecting system using an $18 \mathrm{~F}$ amplatz sheath, and a catheter was placed in the ureter for further irrigation with saline or contrast medium. For initial training with an easy access, a standard guide-wire was inserted in the ureter through the renal parenchyma. The kidney was punctured with radiographic or ultrasound guidance. Minimally invasive percutaneous nephrolithotomy (MIP) was then tested using the model under radiographic or ultrasound guidance. The model was then evaluated in MIP training courses, which are regularly held at The Hannover Medical School.

Results: All trainees were urologists with experience in endourologic surgery but lacked practice in PRS. In conclusion, all 36 participants attained access to the collecting system using models with readily placed guide-wires. Subsequently, PRS was successful in all cases. Percutaneous puncture under ultrasound guidance and following intrarenal surgery was successful in $30(83.3 \%)$ cases. Therefore, all participants rated the model useful for simulating percutaneous renal surgery.

Conclusions: This new porcine kidney model is easy to build and is made cost effective by using readily available material. Moreover, it provides realistic and reproducible training model for PRS. The "organ" model mimics the retroperitoneum by having a full-thickness skin flap with a layer of subcutaneous fatty tissue.
\end{abstract}

Key words: kidney; minimally invasive surgical procedures; percutaneous nephrolithotomy animal models; kidney calculi

Int Braz J Urol. 2011; 37: 388-394

\section{INTRODUCTION}

The increasing incidence and prevalence of urolithiasis in Germany throughout the last decades (1) grew to be a socioeconomic burden with regard to diagnostics and treatment. Thus, new challenges in diagnostic and therapy have to be addressed. A modern treatment of a stone disease has to meet several requirements: It has to be fast and effective, providing a high stone free rate with minimal perioperative morbidity along with a low re-intervention rate. Due to diameter, the number and location of urinary tract stones, mostly ESWL, ureterorenoscopy and percutaneous nephrolithotomy (PCNL) are recommended in the current urologic guidelines (e.g. EAU, AUA Guidelines). The use of percutaneous nephrolithotomy was developed in the 1970s as an alternative to open surgery, but with the introduction of the ESWL in the early 80 s PCNL became less popular. This technical evolution, as well as, the improvement in the field of semirigid and flexible ureterorenoscopy during the following three decades, has dramatically changed the operative management of urolithiasis. Nevertheless, PCNL was recommended for large renal calculi. Although, PCNL has been accepted 
to be safe and effective, it also has significant perioperative morbidity $(2,3)$. Therefore, urologist minimized their PCNL instruments in the past decade so to be less invasive and moreover reduce therapy-associated morbidity. This led to the development of new minimized instruments, where less invasive procedures such as Mini-PCNL (miniaturized PCNL) (4) and MIP (minimally invasive percutaneous nephrolithotomy) $(5,6)$ take part. These techniques showed advantages in terms of shorter hospital stay and reduced postoperative pain while maintaining the high stone free rates of former PCNL (7). These improvements in the field of percutaneous surgery and the critical evaluation and discussion of the results of ESWL and ureterorensocopy with respect to stone free rates and complications led to a rising acceptance of minimally invasive percutaneous procedures throughout the previous years. Therefore, an increasing number of these procedures require sufficient structured training opportunities to maintain surgical effectiveness, with respect to stone free rates and patient safety.

Although miniaturization decreased the perioperative morbidity, percutaneous renal surgery is still one of the most advanced techniques in modern endourology. Frequently, urologists complain of lack of training experience due to scarce training facilities and high cost organ models. Consequently, operative expertise is mostly acquired in the operating theatre. Analysis of structured learning curve of percutaneous nephrolithotomy suggested that basic skills are achieved after $>20$ procedures, surgical competence is achieved after 60 cases and surgical excellence after $>100$ cases (8-10).

An ex-vivo PCNL organ training model described by Zhang et al. (11) was modified in significant aspects. It was then evaluated during MIP training courses at The Hannover Medical School, and has proven efficiency in various teaching sessions taken place in our own department, in addition to the training courses held for urologists. The model allows training of multiple steps of percutaneous renal surgery such as; renal puncture, tract dilation, access sheath introduction, intrarenal endoscopic exploration and further intrarenal sur- gery. It also provides a sufficient tool to overcome the initial learning curve of the first 10 procedures.

\section{MATERIAL AND METHODS}

The PCNL kidney model was built using a porcine kidney, a porcine full thickness skin flap with subcutaneous tissue, an indwelling catheter, artificial renal calculi with a maximum diameter of 5 millimeters and a standard plastic tray.

The kidneys and skin flaps were obtained from freshly slaughtered adult pigs. For ureter preparation and catheterization, a minimum length of $10 \mathrm{~cm}$ ureter was preserved during kidney extraction (Figure-1). The full-thickness skin flap with subcutaneous tissue, in which was harvested from the abdominal wall of adult pigs, was approximately $5-6 \mathrm{~cm}$ in thickness and $40-50 \mathrm{~cm}$ in diameter, enough to cover the entire kidney (Figure-2).

First, the ureter was dissected and intubated with an 18F amplatz sheath (Karl Storz, Tuttlingen, Germany). Afterwards, multiple artificial calculi were placed in the collecting system through the amplatz sheath (Figure-3). The ureter was catheterized for further irrigation with saline or contrast medium using a $12 \mathrm{~F}$ indwelling catheter and then ligated with commercially available sewing material (Figure-4). For the initial training experience, a standard guide wire was inserted through the ureter and diverted bluntly through renal parenchyma providing an easy access to the collecting system with the single-step dilatation system (Karl Storz, Tuttlingen, Germany). The kidney was placed in the center of the skin flap, ensuring that the convex part of the kidney was attached to the fold of the skin flap (Figure-2). Next, the optional guide wire was placed through the skin flap. The kidney was coated with ultrasound gel, to assure sufficient ultrasound visualization during puncture. Finally, the skin wrap was sealed with a running suture of cotton sewing material, and placed into the plastic trays. It is important to note that point out, prepared organ model was not frozen in order to maintain the natural characteristics of the tissue during the surgical training sessions, in contrast to previous descriptions (11). 

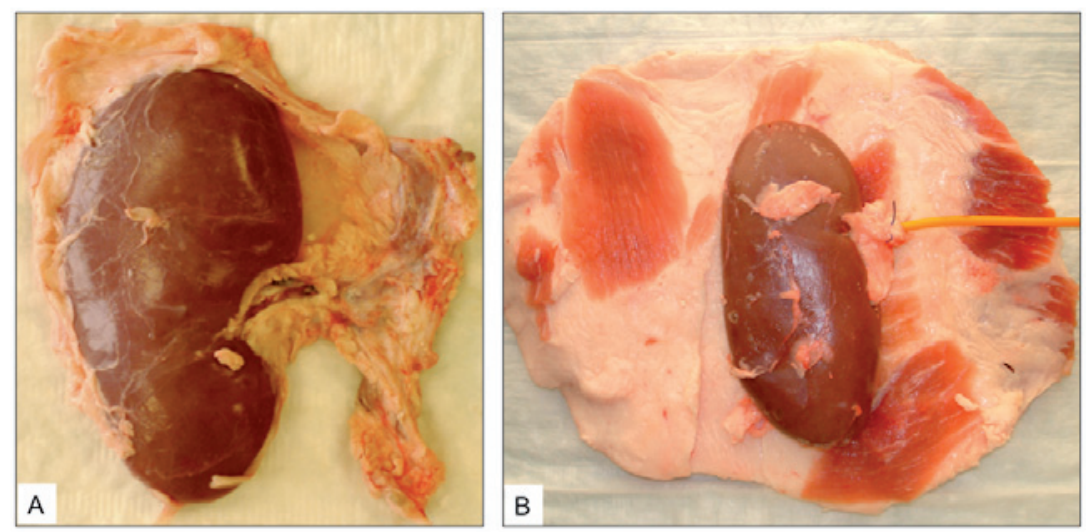

Figure 1 - Freshly harvested porcine kidney (A) and full thickness skin flap used to cover the kidney (B).
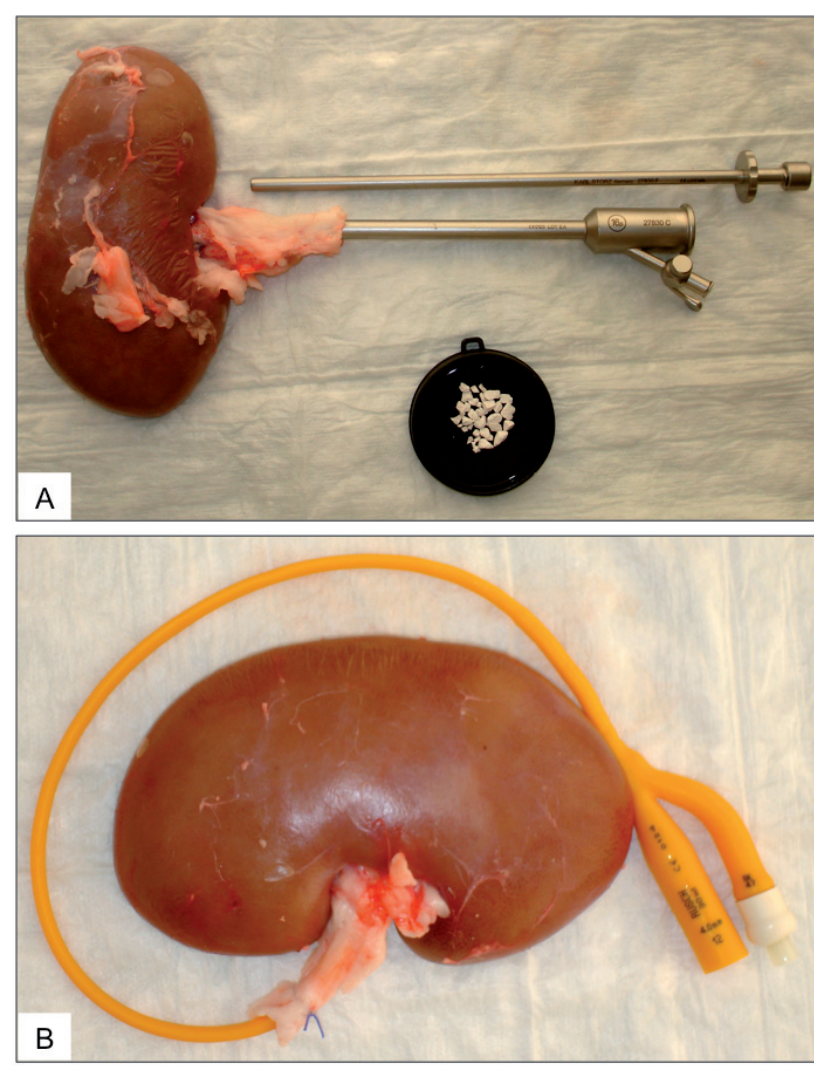

Figure 2 - Placement of artificial calculi into the renal pelvis (A) and catheterized renal pelvis for saline or dye irrigation $(B)$.

The model was applied to percutaneous renal surgery training under radiologic or ultrasound guidance. Either the X-ray unit (Philips Uro Diagnost MRF, Netherlands) or the ultrasound unit (BK Medical, Falcon Ultrasound
Scanner Type 2101, Copenhagen, Denmark) with a 3-6 $\mathrm{MHz}$ probe (BK Medical Type 8803, Copenhagen, Denmark) was used during the procedure. Also, a radiologic contrast medium or normal saline was injected through the indwelling catheter to produce visual images, in order to achieve sufficient artificial hydronephrosis for percutaneous renal puncture. Next, various surgical steps of PRS were performed such as; puncturing, guide-wire placement, single step dilation, insertion of the miniaturized $18 \mathrm{~F} \mathrm{Am-}$ platz sheath (Karl Storz, Tuttlingen, Germany), introduction of a $12 \mathrm{~F}$ nephroscope (Karl Storz, Tuttlingen, Germany), intrarenal exploration, and minimally invasive percutaneous nephrolithotomy (MIP). The organ model was than evaluated in MIP training courses, which are held in our department with basic questionnaire in terms of successful puncture of the collecting system, access to the renal pelvis and subsequent intrarenal surgery. Moreover, the preexisting surgical experience in the field of percutaneous renal surgery of the participating trainees and the personal perception of this model were also evaluated as not useful, undetermined or helpful.

\section{RESULTS}

Based on the previously described organ model (11) a total number of 6 porcine kidneys were necessary to improve and modify this model for percutaneous renal surgery. The preparation of one single organ model acquired 10-15 minutes time, until it was ready for a percutaneous training session.

Altogether, 36 urologists attended 6 urologic training courses for percutaneous renal surgery at the affiliated hospitals. All 36 participants were known to have experience in urologic endoscopic surgery (defined as ureteroscopy, renoscopy) yet no experience in the field of percutaneous renal surgery (defined as minimally invasive percutaneous nephrolithotomy (MIP), percutaneous 

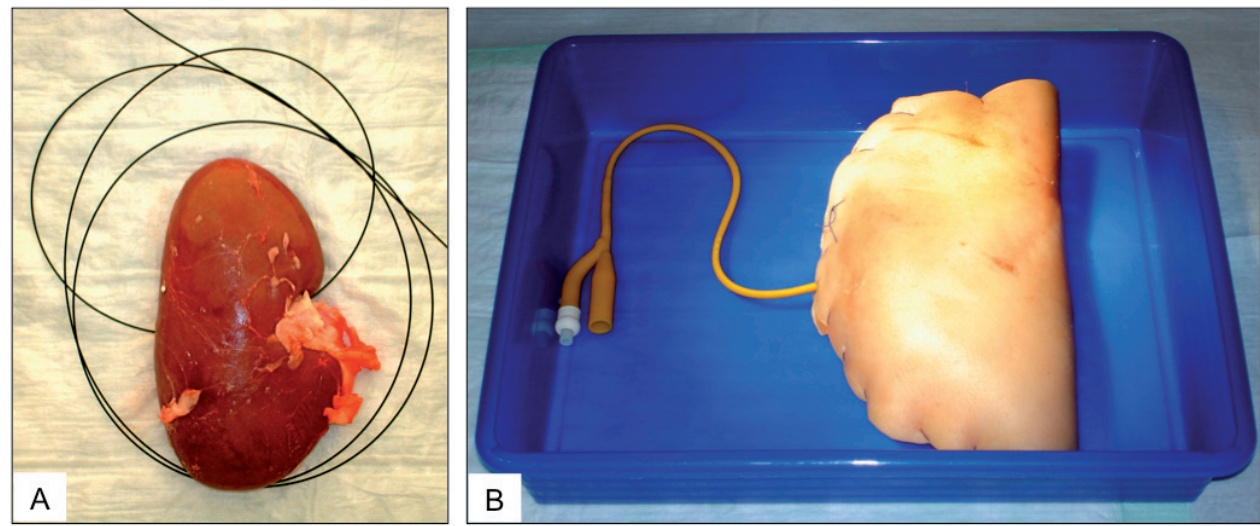

Figure 3 - Guide-wire for easy access to the renal pelvis (A) and wrapped organ model, closed by running sutures, in the plastic tray for subsequent training (B).
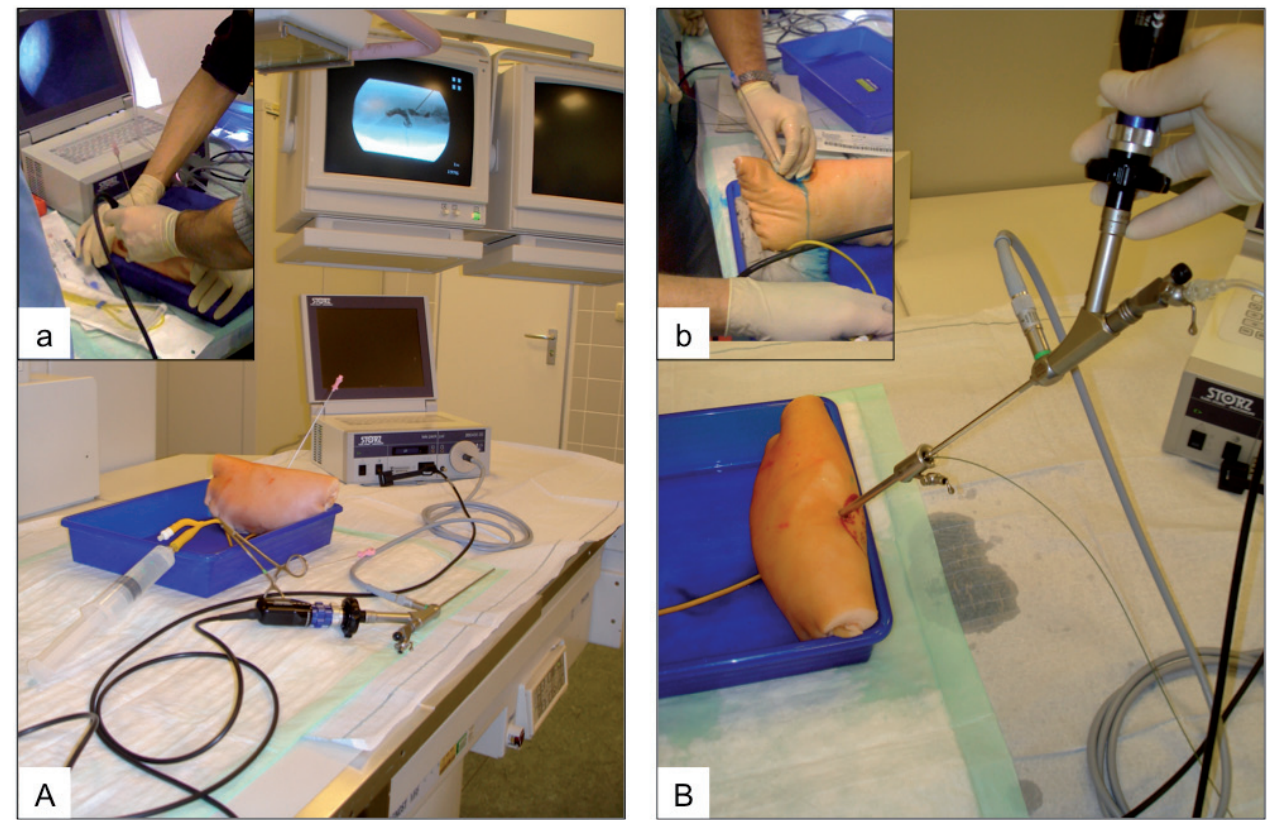

Figure 4 - Puncture of the renal pelvis (A) and subsequent intrarenal surgery (B). Alternate ultrasound guided puncture (a) and verification of successful access to the renal pelvis by blue dye irrigation is depicted (b).

nephrolithotomy (PCNL)). All 36 (100\%) trainees were able to access the renal pelvis using the designed model, readily prepared with a guide wire. Furthermore, percutaneous surgery (MIP) was successful in all cases through this predefined access. $30(83.3 \%)$ participants managed to achieve access to the collecting system by ultrasound or radiographic guidance. If surgical access was achieved, subsequent percutaneous transrenal surgery (MIP) was successful in 30 cases $(100 \%)$. All participants practiced the percutaneous handson manipulations on this model under the direct guidance and surveillance of three experienced endourologists. By the end of this course, all attendants rated the porcine kidney model for simulation of percutaneous renal surgery as "helpful". 
The low cost for this organ model was the result of use of inexpensive ureteral catheters for irrigation of the renal pelvis during percutaneous surgery, as well as, the use of standard sewing material. The use of reusable material and instruments improved the cost-value relation of this model. In summary, the total cost accounted approximately $10 €$ for each prepared organ model. Nonetheless to be considered, that initial cost for the described reusable instruments in animal use only may occur establishing an organ model training program for percutaneous renal surgery.

In conclusion, this percutaneous renal access and other intrarenal procedures proved feasible and practical under radiographic or ultrasound guidance.

\section{DISCUSSION}

Learning percutaneous renal surgery is still demanding, although several virtual and biologic models have been published. But only virtual or laboratory training of percutaneous renal surgery provides the opportunity to overcome the initial learning curve. Thus, training models have to meet several requirements. These models must be cost effective, easy and fast to prepare with commonly available material and organs. They need to be realistic, provide the feeling of human tissue and simulate the retroperitoneal anatomy. Finally, they should provide easy access to the renal pelvis for subsequent renal surgery, along with a high success rate under training conditions. To address these challenging requirements, several training models have been published to date, consisting of virtual computer-based non-biologic training and organ-based models using porcine kidneys.

A nonbiologic computer-based simulator PERCMentor (Simbionix, Lod, Israel) has been published by Knudsen et al. in 2006, providing virtual reality skills. This might allow trainees to develop the basic skills necessary to perform percutaneous access to the renal collecting system (12). However, expenses of this sort of training system are unclear for software and hardware costs, addition to time-consuming labor-intensive training of urologists. The advantage of biologic models is the "tissue feeling", an imitation of human tissue while allowing a great variety of procedures of intrarenal surgery (13). To date 6 reports have been published providing feasible biologic models using porcine kidneys for percutaneous renal surgery training. Porcine kidneys have been wrapped in a foam layer, embedded in silicon, enclosed in chicken carcasses and in porcine thoracic/abdominal walls. Radiologic and ultrasound guidance were applied for percutaneous renal access guidance (13-17).

A model published by Zhang et al. in 2008 was primarily the inspiration of building our own (11). This ex-vivo porcine kidney training model was significantly improved in meeting criterions of cost effectiveness, preparation, and simplified access to the collecting system in the initial training phase and therefore, concluding success rates in percutaneous renal surgery. Not to mention, improved cost effectiveness was achieved by diminishing the use of expensive disposable materials. Moreover, the ureteral catheter was replaced by a $12 \mathrm{~F}$ indwelling catheter and reusable plastic trays were used instead of wooden boards. The use of these plastic trays not only improves the hygiene, but it allows the collection of the saline irrigation during the procedures. The full-thickness skin was replaced by porcine entire abdominal wall of adult pigs including the subcutaneous tissue, providing enhanced ultrasound visualization for subsequent puncture, as well as improvement in realistic retroperitoneal circumstances with regard to consistency and resistance of the skin. This setting allows a realistic training of puncturing, single step tract dilatation, amplatz sheath introduction and subsequent intrarenal surgery. The porcine kidney anatomy is similar to that of humans, facilitating intrarenal endoscopic practice. The transureteral introduction of renal calculi through the $18 \mathrm{~F}$ amplatz sheath into the renal pelvis significantly eased the preparation of the organ model.

Although the described organ model is cost-effective, other issues must be considered such as hygiene standards and policies, instrument sterilization and use of disposables during training. 
In contrast to previous descriptions, our experience using the depicted organ model supports that neither an immediate irrigation following the kidney harvest (15), nor the refrigeration of the organ model (11) is required to maintain the rigidity of the kidney tissue. It was demonstrated high success rates for optimal puncture of the collecting system, subsequent tract dilatation followed by intrarenal surgery. Thus, this organ model can easily be prepared hours before a training session, reducing labor and preparation while retaining realistic scenario for training.

In contrast to other models $(13,14)$, the kidney is not fixed to the surrounding structures in the skin-flap compartment, providing a sufficient and realistic mobility of the kidney, mimicking in-vivo circumstances. Comparing to organ models with porcine kidneys embedded in chicken carcasses (15), this model sufficiently avoids interferences in X-ray or ultrasound based puncture, usually caused by the chicken skeleton. Thus, improvement of success level in the initial training period was up to 10 percutaneous interventions in particular. This ex-vivo model lacks the simulation of ribs, simulating realistic conditions as in human anatomy. This could be developed to some extent by using part of the thoracic wall and superficial soft tissue to give an even better practice environment (17). Such model might improve the training results creating further advanced urologic surgeons with basic skills in percutaneous surgery.

All attending trainees were urologists (36/100\%) without experience in percutaneous renal surgery while previously experienced in transurethral endourology. It is intriguing, whether the reported high success rate accessing the renal pelvis was due to the easy surgical access by a readily placed guide wire, or by pre-existing surgical skills in minimally invasive endourology. This organ model was used in 6 training sessions with 6 participants. An initial 90 minute didatic lesson with basic knowledge of percutaneous renal surgery was followed by a 3-hour hands-on-training at 3 training venues simultaneously. All trainees were personally instructed by three certified urologists with the opportunity of puncture practice, tract dilatation and percutaneous surgery at their own organ models. This setting allowed individual instructions given to all trainees, to guarantee the reported high success rate. Moreover, the individual training with personal porcine organ models led to the acceptance of the model, in which was demonstrated by the evaluations.

\section{CONCLUSIONS}

This porcine kidney model previously described is simple, cost effective and easy to prepare with reusable material and instruments. It mimics the natural circumstance, and provides realistic and reproducible practice for percutaneous renal surgery in the training laboratory. Furthermore, it provides incremental training opportunities for trainees with various skills in the field of percutaneous surgery with an easier wired access to the renal pelvis for the initial training period. The majority of the participating trainees evaluated this organ model as "helpful". Hence, it is believed that this model will become an integral part of structured training for minimally invasive percutaneous nephrolithomy (MIP) "in the near future".

\section{ABBREVIATIONS \\ PRS Percutaneous renal surgery \\ MIP Minimally invasive percutaneous nephro- lithotomy \\ ESWL Extracorporal shockwave lithotripsy \\ PCNL Percutaneous nephrolithotomy \\ EAU European Association of Urology \\ AUA American Association of Urology \\ F French}

\section{CONFLICT OF INTEREST}

None declared.

\section{REFERENCES}

1. Hesse A, Brändle E, Wilbert D, Köhrmann KU, Alken P: Study on the prevalence and incidence of urolithiasis in Germany comparing the years 1979 vs. 2000. Eur Urol. 2003; 44: 709-13.

2. Michel MS, Trojan L, Rassweiler JJ: Complications 
in percutaneous nephrolithotomy. Eur Urol. 2007; 51: 899-906; discussion 906.

3. Osman M, Wendt-Nordahl G, Heger K, Michel MS, Alken P, Knoll T: Percutaneous nephrolithotomy with ultrasonography-guided renal access: experience from over 300 cases. BJU Int. 2005; 96: 875-8.

4. Lahme S, Zimmermanns V, Hochmuth A, Janitzki V: Minimally invasive PCNL (mini-perc). Alternative treatment modality or replacement of conventional PCNL?. Urologe A. 2008; 47: 563-8.

5. Nagele U, Knoll T, Schilling D, Michel MS, Stenzl A: Lower pole calyceal stones. Urologe A. 2008; 47: 875-84.

6. Nagele U, Schilling D, Anastasiadis AG, Walcher U, Sievert KD, Merseburger AS, et al.: Minimally invasive percutaneous nephrolitholapaxy (MIP). Urologe A. 2008; 47: 1066, 1068-73.

7. Knoll T, Wezel F, Michel MS, Honeck P, WendtNordahl G: Do patients benefit from miniaturized tubeless percutaneous nephrolithotomy? A comparative prospective study. J Endourol. 2010; 24: 10759.

8. Allen D, O'Brien T, Tiptaft R, Glass J: Defining the learning curve for percutaneous nephrolithotomy. J Endourol. 2005; 19: 279-82.

9. de la Rosette JJ, Laguna MP, Rassweiler JJ, Conort $\mathrm{P}$ : Training in percutaneous nephrolithotomy--a critical review. Eur Urol. 2008; 54: 994-1001.

10. Tanriverdi O, Boylu U, Kendirci M, Kadihasanoglu M, Horasanli K, Miroglu C: The learning curve in the training of percutaneous nephrolithotomy. Eur Urol. 2007; 52: 206-11.

11. Zhang Y, Ou TW, Jia JG, Gao W, Cui X, Wu JT, et al.: Novel biologic model for percutaneous renal surgery learning and training in the laboratory. Urology. 2008; 72: 513-6.

12. Knudsen BE, Matsumoto ED, Chew BH, Johnson B, Margulis V, Cadeddu JA, et al.: A randomized, con- trolled, prospective study validating the acquisition of percutaneous renal collecting system access skills using a computer based hybrid virtual reality surgical simulator: phase I. J Urol. 2006; 176: 2173-8.

13. Strohmaier WL, Giese A: Ex vivo training model for percutaneous renal surgery. Urol Res. 2005; 33: 1913.

14. Earp PP: Percutaneous renal surgery--new model for learning and training. Int Braz J Urol. 2003; 29: 1514.

15. Häcker A, Wendt-Nordahl G, Honeck P, Michel MS, Alken P, Knoll T: A biological model to teach percutaneous nephrolithotomy technique with ultrasoundand fluoroscopy-guided access. J Endourol. 2007; 21: $545-50$.

16. Hammond L, Ketchum J, Schwartz BF: A new approach to urology training: a laboratory model for percutaneous nephrolithotomy. J Urol. 2004; 172: 1950-2.

17. Strohmaier WL, Giese A: Improved ex vivo training model for percutaneous renal surgery. Urol Res. 2009; 37: 107-10.

Accepted after revision: December 15, 2010

\author{
Correspondence address: \\ Dr. Florian Imkamp \\ Clinic for Urology and Urologic Oncology \\ Hannover Medical School \\ Carl-Neuberg-Str. 1 \\ Hannover, 30625, Germany \\ Fax: + $49511532-3481$ \\ E-mailimkamp.florian@mh-hannover.de
}

